\title{
Nutrition and health in the developing world: the Caribbean experience
}

\author{
BY KNOX E. HAGLEY \\ Department of Social and Preventive Medicine, University of the West Indies, \\ Mona, Kingston 7, Jamaica
}

Caribbean countries are in transition. Changes, widespread and rapid, have been taking place in the economic and social arenas, at the same time that fundamental political changes have been pursued and enacted. Changes in the field of health have been no less dramatic and in a sense, they do reflect, if not mirror, the changes in the other arenas. This state of affairs is true of virtually all developing countries. Indeed, changes are implicitly implied in the use of the term developing.

Changes in the health arena in Caribbean countries have been both rapid and profound and have brought about an epidemiological transition which has shifted the infectious or communicable diseases from centre stage and replaced them with the chronic non-communicable diseases within the relatively short time frame of a mere two-three decades. In all of this the contribution of nutrition has been outstanding.

The concept that food is necessary, not only for the provision of energy but also for the maintenance of health has been long held. The use of food to combat disease, whatever its nature or origin, has been a fundamental strategy of all peoples of all times.

The subsequent link between the development of disease and a specific nutritional deficiency, therefore, would have probably occasioned no surprise but would have served as a dramatic example of the role of nutrition in the maintenance of health. This must have been so at the time when it was shown by a basic, but beautiful example of epidemiology at work, that a fearful bleeding disorder among English sailors of centuries ago could have been both cured and prevented by merely ensuring an adequate supply of fruits in the diet. The effectiveness of the lowly lime to prevent the disorder which subsequently became known as scurvy no doubt served to enhance the growing recognition of the value of food.

In more recent times, the recognition of undernutrition of a more general nature, usually referred to as malnutrition, as a cause of much ill-health further underlined the importance of food in general and its nutrients in particular in the maintenance of a healthy body. Much of the knowledge gained about this relationship has been garnered during this century, primarily in developing countries, many of which have been burdened with the problems of malnutrition. In this regard, the Caribbean experience has been one of some magnitude.

The understanding that excessive intake of food can also lead to ill-health, from a variety of diseases, was much later in coming and, in the developing countries, is a relatively recent experience. In Caribbean countries, this form of malnutrition is already very much in evidence and is now compromising the health of nationals at a time when pockets of the more conventional malnutrition in children can still be identified (Sinha, 1988).

Small wonder then that the Caribbean region has been the scene of an almost continuous epidemiological transition for the greater part of this century. Events in the English-speaking countries portray the breadth and depth of this transition and, indeed, they do epitomize the experience of the Caribbean region as a whole. 
The massive mortality, which had been inflicted for several centuries on these countries by the infectious diseases, lessened considerably when yellow fever was brought under control in the early part of the 20th century and the incidence of malaria fever steadily reduced until the final eradication of indigenous malaria in the early 1960s. Nevertheless, infant mortality rates were still quite high around the middle of the century when rates for the English-speaking countries averaged approximately $85 / 1000$, with some countries registering rates of well over 100/1000 (Pan American Health Organization, 1964).

It soon became obvious that, although pneumonia and gastroenteritis were frequently listed as the causes of death, existing malnutrition had undermined the health of large numbers of the children who had perished. Furthermore, malnutrition was also taking its toll on large numbers of children in the Caribbean communities. This state of affairs demanded urgent and concerted efforts to improve the levels of nutrition among the children.

The problem of malnutrition had also attracted medical academia which had only recently made its entry into the anglophone countries of the region. It was John Waterlow, who had pioneered Caribbean research into malnutrition. He began his work on the children's ward of the hospital of the recently established University College of the West Indies and later led his many teams at the Medical Research Unit which was set up on the University Campus and which later became the Tropical Metabolism Research Unit. Over the years distinguished researchers at that unit have continued to make outstanding and invaluable contributions to the understanding and treatment of malnutrition among children.

Alas, the search for solutions did not always lead to a clear understanding of the issues and in their efforts to account for the varied clinical picture of malnutrition, researchers in the Caribbean and elsewhere resorted to compartmentalization of disorders, only to find that marasmus and kwashiorkor were not as distinct as they were made out to be and, quite naturally, the term marasmus-kwashiorkor came into play, but that too soon proved to be inadequate. Protein-energy malnutrition possesses the virtue of 'telling it like it is' and therein lies its attraction. The Tropical Metabolism Research Unit in Jamaica has continued to make its presence felt in the field of nutrition, as exemplified by work at the unit in recent years (Golden \& Ramdath, 1987) which has provided fresh and interesting insights into the pathogenesis of kwashiorkor, a condition that continues to baffle and excite nutritionists at all levels.

Improvement in the socio-economic status and education levels of the main mass of Caribbean peoples, together with specific educational and other activities, led to notable improvements in the nutritional status of children, to the extent that by the mid-1970s undernutrition was no longer a widespread problem (Sinha, 1988). This was no mean feat in countries whose per capita income had increased only marginally during those years.

At the same time, improved levels of sanitation and other public health activities, rising rates of immunization (most English-speaking Caribbean countries now boast a coverage of about $90 \%$ for most of the childhood diseases preventable by immunization), enhancement of therapeutic capability through the advent of antibiotics have all played important roles in the control of the infectious diseases. The net effect of all of these happenings was steep falls in infant mortality rates in the English-speaking Caribbean which now average $22 / 1000$, with rates in many of the islands in the $16-20 / 1000$ range. 
Experience in the Dutch and French-speaking islands (with the sad exception of Haiti) as well as the Spanish-speaking countries is similar.

The considerable lowering of infant mortality rates, the steady though slow falls in birth rates and mass migration of young people in the 1950s and 1960 s led to rapid aging of the population. Indeed, by 1985 the Caribbean region had gained the reputation of being the region in the developing world with the highest percentage of the population made up of persons aged 55 years and over (Kinsella, 1988). Life expectancy now averages 68 years among males and 71 years among females.

These changes resulted in a substantial increase in the target population for the chronic non-communicable diseases and this was soon to be reflected in mortality data from the various countries. It became obvious by the latter part of the sixth decade of this century that a shift was occurring among the leading causes of death. For instance, although heart disease had maintained its number one position, the aetiology of cardiac pathology was undergoing changes, in that hypertension was making an increasingly larger contribution and by the seventh decade had become the main cause of heart disease in the region.

Over the past 25 years the three leading causes of mortality in the region have been the same as those seen at the top of mortality tables in the developed world: cardiac disease, cerebrovascular disease and malignant neoplasms. The presence of cerebrovascular disease among that group underlines the contribution of hypertension to mortality. This condition is the commonest of the non-communicable diseases and population-based studies have revealed its presence in at least $30 \%$ of adults aged 35 years and over (Hagley, 1990).

Diabetes mellitus, another common disorder, is present in at least $12 \%$ of adults in that same age-group. In keeping with the experience of other countries, this disorder is seen with considerably greater frequency among those of East-Indian origin but is still considered to be rare among the Amerindians, the original inhabitants of the islands, who have retained their native way of life. An interesting aspect of diabetes, and one which is cause for considerable concern, is the rapid rise in prevalence rates of the disorder. Population-based studies in Jamaica, Trinidad, Tobago and Barbados have shown an increase in rates of approximately $300 \%$ over the past three decades. The contribution of diabetes to mortality has increased considerably and, although mortality lists have been slow to recognize its contribution, pathological data obtained from ten of the English-speaking countries over 12 years ago had revealed that cardiovascular disease and diabetes were responsible for almost $40 \%$ of total mortality in these countries (McGlashan, 1982).

Coronary artery disease, which was a rare clinical entity and distinctly uncommon pathological finding until the early 1960 s, seemed to gallop thereafter and is now a major cause of cardiac mortality in the more developed of the countries and no longer rare in the smaller, less-developed territories.

Considerable changes have also taken place in the cancer arena over the past three decades. The numbers have obviously grown but a finding of particular interest to nutritionists is the changing pattern of malignancies seen. Gastric cancer, traditionally linked to certain nutritional and dietary practices, was previously the leading cancer in both sexes but now occupies the third spot among both males and females in most countries. On the other hand, cancer of the colon and rectum which was rarely seen in the 1950 s and 1960 s is now rated the fourth-leading malignancy in both sexes. Among 
males, cancers of the prostate and lung are vying for first place and among females, the increasingly frequent breast cancer is rivalling cancer of the uterine cervix for first spot.

A number of factors, undoubtedly, have been contributing to this tremendous surge in prevalence of the chronic non-communicable diseases which Caribbean countries have experienced over the past four decades. It is obvious, however, that the leading diseases, in terms of both mortality and morbidity, belong to the group recognized as nutritionbased disorders.

Data analysis of food intake in the English-speaking Caribbean has shown that, in general, there has been a progressive decrease in consumption of complex carbohydrate but an increase in the use of simple sugars, and an increase in the intake of animal fats. This Westernization of Caribbean palates should come as no surprise because the region is geographically and otherwise at risk for a style of living which is so pervasive anyway.

The Caribbean also provides a good example of the way in which the economy of a country can influence nutrition and dietary practices. A comparison between Barbados and Guyana bears out this point (Sinha \& McIntosh, 1992). Between 1960 and 1982, the Barbados economy was strong and experienced an average growth of gross domestic product (GDP) of $4.5 \%$. In that period daily energy availability per capita increased from $10 \cdot 8$ to $13 \cdot 1 \mathrm{MJ}$ (i.e. $30 \%$ ) above its average requirement. Of further interest was the finding that the contribution of complex carbohydrate to energy intake had decreased from 52 to $41 \%$, whereas contribution of animal fats had climbed and contribution of total fat rose from 20 to $29 \%$. Guyana in that same period had experienced a GDP growth of only $1.7 \%$. The average energy intake per capita had risen only slightly from 9.6 to $9.8 \mathrm{MJ}$, the contribution of complex carbohydrate had risen from an already impressive $62 \%$ to $66 \%$ but energy from total fat had declined from 18 to $14 \%$. A finding worthy of note, and one which must be of particular interest to politicians, is the fact that during that period (1960-82) $60 \%$ of energy consumed by Barbadians was imported, whereas the contribution of imported food to total energy consumed by the Guyanese had fallen from $34 \%$ in $1964-6$ to $18 \%$ in $1986-8$.

Comparison of another kind between these two countries provides the type of information which excites clinicians, pathologists and epidemiologists alike because data from Barbados and Guyana reveal notable differences between the two countries in the contribution of the various chronic non-communicable diseases to mortality. Crude mortality rates of diabetes, hypertension, coronary artery disease and strokes are all considerably higher in Barbados and this is also true of colon and breast cancers (Sinha \& McIntosh, 1992). The fact is that the nutrition-related chronic diseases are taking a toll on the health of the Barbadian community which is considerably greater than that experienced by the Guyanese community.

The prevalence of obesity, that most visible of the nutrition-based disorders, lends further and considerable support for attributing to nutrition, a major role in the pathogenesis of the leading disorders. Nutrition surveys in various English-speaking Caribbean countries have shown high and rising prevalence rates of the disorder among middle-aged females to the extent that at present approximately one in two females aged 40 years or over is obese. The disorder is also increasing among adult males and there is evidence also that obesity is already a significant finding among adolescent females (Ramsey et al. 1986). It is patently obvious that while Caribbean countries were expending efforts to reduce undernutrition among children, overnutrition among adults was not only present but growing. It is also now evident that, apart from excessive intake 
of energy among adults, other changes have been taking place in the nutrition and dietary habits of Caribbean peoples.

Data for assessing the direct impact of obesity on mortality in the Caribbean region are not available but, bearing in mind that the disorder is a high risk factor for diabetes, hypertension and coronary artery disease and noting that it is also a risk factor for breast and other cancers, the single act of reducing prevalence of obesity has the potential to lessen, to a considerable degree, the contribution of the chronic diseases to mortality. This is a lesson that both developed and developing countries must not fail to heed.

\section{REFERENCES}

Golden, M. \& Ramdath, D. (1987). Free radicals in the pathogenesis of kwashiorkor. Proceedings of the Nutrition Society 46, 53-68.

Hagley, K. E. (1990). Chronic disease: An overview of the problem. Cajanus 23, 8-13.

Kinsella, K. G. (1988). Aging in the Third World. Center for International Research, Washington DC, Staff Paper no. 235, pp. 1-8. Washington, D.C.: Center for International Research.

McGlashan, N. D. (1982). Causes of death in ten English-speaking Caribbean countries. Bulletin of the Pan American Health Organization 16, 212-223.

Pan American Health Organization (1961-1964). Health Conditions in the Americas. PAHO Scientific Publication no. 138, pp. 38-49. Washington, DC: PAHO.

Ramsey, F., Demas, N. \& Trotter, P. (1986). The National Health and Nutrition Survey of Barbados, 1981. Kingston, Jamaica: Caribbean Food and Nutrition Institute.

Sinha, D. (1988). Nutrition in the English-speaking Caribbean; a brief review of the changes in the last three decades. Cajanus 21, 113-132.

Sinha, D. \& McIntosh, C. (1993). Changing nutritional patterns and their implications for food availability in the Caribbean. Food and Nutrition Bulletin. Rome: United Nations University (In the Press). 